\title{
Exploration of pro-oxidant and antioxidant activities of the flavonoid myricetin
}

\author{
Vladimir Chobot, Franz Hadacek
}

Department of Chemical Ecology and Ecosystem Research, Faculty of Life Sciences, University of Vienna, Vienna, Austria

Objectives: Flavonoids are ubiquitous phenolic plant metabolites. Many of them are well known for their proand antioxidant properties. Myricetin has been reported to be either a potent antioxidant or a pro-oxidant depending on the conditions. The reaction conditions for the pro- and antioxidant activities were therefore investigated using variations of the deoxyribose degradation assay systems.

Methods: The deoxyribose degradation assay systems were conducted as follows; $\mathrm{H}_{2} \mathrm{O}_{2} / \mathrm{Fe}$ "II/ ascorbic acid, $\mathrm{H}_{2} \mathrm{O}_{2} / \mathrm{Fe}^{\prime \prime \prime}$, $\mathrm{Fe} \mathrm{e}^{\prime \prime \prime} /$ ascorbic acid, and $\mathrm{Fe}$ lII. Each system was carried out in two variants, $\mathrm{FeCl}_{3}$ (iron ions added as $\mathrm{FeCl}_{3}$ ) and FeEDTA (iron added in complex with ethylenediaminetetraacetic acid).

Results: When ascorbic acid was present, myricetin showed antioxidant properties, especially when it occurred in complex with iron. In ascorbic acid-free systems, pro-oxidant activities prevailed, which where enhanced if iron was in complex with EDTA.

Discussion: Myricetin's antioxidant activity depends on both the reactive oxygen species (ROS) scavenging and iron ions chelation properties. The pro-oxidative properties are caused by reduction of molecular oxygen to ROS and iron(III) to iron(II). Myricetin is able to substitute for ascorbic acid albeit less efficiently.

Keywords: Flavonoids, Free radical scavengers, Hydroxyl radical, Iron chelating agents, Reactive oxygen species

\section{Introduction}

Flavonoids are widely occurring plant phenols. Their effects on human health have been described repeatedly, ${ }^{1}$ but the actual benefit to the producing plant is still controversial, although various explanations have been offered. ${ }^{2}$ One such advantage may be redox homeostasis regulation by scavenging or generation of reactive oxygen species (ROS). ${ }^{3,4}$ ROS are better known for causing cytoxicity, but also may affect various cellular signal cascades and metabolic processes. ${ }^{5}$ ROS are required in specific low concentrations for normal cell functioning. ${ }^{3,5,6}$ Many plant secondary metabolites act as antioxidants and pro-oxidants and can affect ROS concentrations depending on the reaction conditions. ${ }^{3,7}$ In aromatic compounds such as flavonoids, antioxidant activities depend on the redox properties of the phenolic hydroxyl groups, especially on those of the catechol or pyrogallol moieties, which are easily oxidized. ${ }^{8,9}$ Reduction of molecular oxygen to superoxide anion radical and iron(III) to iron(II) ions can cause pro-oxidant activities. ${ }^{9}$ Many of these redox reactions, however, are

Correspondence to: Vladimir Chobot, Department of Chemical Ecology and Ecosystem Research, Faculty of Life Sciences, University of Vienna, Althanstraße 14, A-1090 Vienna, Austria.Email: vladimir.chobot@univie.ac.at<smiles>O=c1c(O)c(-c2cc(O)c(O)c(O)c2)oc2cc(O)cc(O)c12</smiles>

Figure 1 Structure of myricetin.

reversible, the specific reaction mixture and conditions resulting in complex equilibria. ${ }^{10,11}$

Myricetin (Fig. 1) serves as a good example of the complexity of this phenonema. ${ }^{12}$ Controversial reports on myricetin's effects, both as an antioxidant and a pro-oxidant, stimulated us to evaluate its redox properties in the deoxyribose degradation assay, which was originally developed to assess test compound reactions with hydroxyl radicals $\left(\mathrm{HO}^{\bullet}\right)$ that are produced by the Fenton reaction: ${ }^{13,14}$

$$
\mathrm{Fe}^{\mathrm{II}}+\mathrm{H}_{2} \mathrm{O}_{2} \rightarrow \mathrm{Fe}^{\mathrm{III}}+\mathrm{HO}^{\bullet}+\mathrm{HO}^{-} \text {(Fenton reaction) }
$$

It utilizes the oxidative degradation of 2-deoxy-Dribose by hydroxyl radicals as the detection mechanism for antioxidative activity. The degradation 
products can be quantified photometrically after reacting with 2-thiobarbituric acid to thiobarbituric acidreactive species (TBARS). ${ }^{11}$

We performed the deoxyribose degradation assay in four systems: ${ }^{11}$

1. $\mathrm{H}_{2} \mathrm{O}_{2} / \mathrm{Fe}^{I I I} /$ ascorbic acid: Hydroxyl radicals are generated by the Fenton reaction after reduction of iron(III) to iron(II) by ascorbic acid. ${ }^{13,14}$ This system shows possible interactions of the tested compound with the Fenton reaction and oxidative degradation of 2-deoxy-D-ribose.

2. $\mathrm{H}_{2} \mathrm{O}_{2} / \mathrm{Fe}^{I I I}$ : If ascorbic acid is omitted from the reaction mixture, the tested compound can start the Fenton reaction by reducing iron(III). ${ }^{15}$ This assay modification detects a possible replacement of ascorbic acid by the test compound in the reaction mixture. The reaction of the tested compound with iron(III) has to be kinetically more favorable than that of hydrogen peroxide.

3. $\mathrm{Fe}^{I I I}$ / ascorbic acid: Ascorbic acid reduces iron(III) to iron(II), which reacts further with molecular oxygen present in the reaction mixture by generating superoxide anion radicals. ${ }^{11,16}$ These dismutate to molecular oxygen and hydrogen peroxide. The superoxide anion radicals may also be reduced to hydrogen peroxide by one electron transfer from ascorbic acid or the tested compound. Iron(III) reduction and hydrogen peroxide formation start the Fenton reaction. This system explores possible interactions of the test compound with hydroxyl radical generation by autoxidation of ascorbic acid.

4. $\mathrm{Fe}^{I I I}$ : In the presence of iron(III) ions, the test compound may be oxidized and, in consequence, iron(III) may be reduced to iron(II) which then reduces molecular oxygen present in the reaction mixture to superoxide anion radical. The latter dismutates into molecular oxygen and hydrogen peroxide which starts the Fenton reaction. ${ }^{11}$

To all these systems, iron(III) is added in two variants, either as $\mathrm{FeCl}_{3}$ or in complex with ethylenediaminetetraacetic acid (EDTA). If iron ions are added as chloride, they can form a direct complex with the test compound ${ }^{13}$ facilitating studying of the test compoundiron complex. An already-formed iron(III)-EDTA complex, however, prevents complex formation of iron with other ligands and allows exploration of the chemistry of the test compound when it is not a ligand of a transition metal. ${ }^{15}$

\section{Materials and methods Chemicals}

Hydrogen peroxide and 2-deoxy-D-ribose were obtained from Fluka (Buchs, Switzerland). All other chemicals used were purchased from Sigma-Aldrich Inc. (St Louis, MO, USA). Water had Milli-Q quality.

\section{Deoxyribose degradation assay}

The various systems and variants of the assays follow procedures of which detailed reaction mechanisms are described elsewhere. ${ }^{11}$ In short, the assays comprise two systems: in one, $\mathrm{H}_{2} \mathrm{O}_{2}$ is added and, in the other, absent. Accordingly, since $\mathrm{H}_{2} \mathrm{O}_{2}$ an important reactant for the Fenton reaction was missing in the second system, additional time was needed to allow oxygen to diffuse into the reaction solution and to be subsequently reduced to ROS. Each system was performed in two sets (ascorbic acid present or absent); the latter set aimed to explore if myricetin can substitute for ascorbic acid. Furthermore, there are two variants: Iron was either added as $\mathrm{FeCl}_{3}\left(\mathrm{FeCl}_{3}\right.$ variant) or as $\mathrm{Fe}^{\mathrm{III}}$ complex with EDTA (FeEDTA variant). Myricetin was dissolved in a dilution series $(2-500 \mu \mathrm{M})$ in an aqueous $\mathrm{KH}_{2} \mathrm{PO}_{4} / \mathrm{KOH}$ buffer solution $(50 \mathrm{mM}, \mathrm{pH} 7.4)$; to $125 \mu \mathrm{l}$ of this solution, $25 \mu \mathrm{l}$ of a $10.4 \mathrm{mM}$ 2-deoxy-D-ribose solution in the same buffer system and $50 \mu$ lof aqueous $\mathrm{FeCl}_{3}$ solution $(50 \mu \mathrm{M})$ were added. In one series, the latter $50 \mu \mathrm{l}$ contained $52 \mu \mathrm{M}$ EDTA dissolved in buffer, which was premixed with the aqueous $\mathrm{FeCl}_{3}$ solution $(1: 1 \mathrm{v} / \mathrm{v})$. In the other series, the EDTA volume was replaced by buffer. Further, $25 \mu 110.0 \mathrm{mM}$ aqueous solution of $\mathrm{H}_{2} \mathrm{O}_{2}$ and $25 \mu \mathrm{l}$ of $1.0 \mathrm{mM}$ ascorbic acid in the buffer were added to start the Fenton reaction in the $\mathrm{H}_{2} \mathrm{O}_{2} / \mathrm{Fe}^{\mathrm{III}} /$ ascorbic acid reaction mixture. In the other deoxyribose degradation assays systems, $\mathrm{H}_{2} \mathrm{O}_{2}$ or ascorbic acid was replaced by the same volume of water or buffer, respectively. TBARS were determined photometrically at $532 \mathrm{~nm}$ after reaction with thiobarbituric acid and subsequent extraction of the pink pigment with 1-butanol. The positive control was the $\mathrm{H}_{2} \mathrm{O}_{2} / \mathrm{Fe}^{\mathrm{III}} /$ ascorbic acid reaction mixture lacking the test compound (100\% TBARS in all systems and variants, determined in each series of the experiments to facilitate result comparability). Blanks contained the full reaction mixtures except for 2-deoxy-D-ribose and were determined in each replicated experiment. Assays were performed in triplicates. The systems containing $\mathrm{H}_{2} \mathrm{O}_{2}$ were evaluated after 1 hour. The systems without $\mathrm{H}_{2} \mathrm{O}_{2}$ were evaluated after 16 hours. The temperature during incubation was $27^{\circ} \mathrm{C}$.

\section{Statistical analysis}

Statgraphics Plus 5.0 (Statistical Graphics Corp., Rockville, MD, USA) was used to perform analyses of variance (ANOVA) with 95\% Duncan's multiple range test. The dose-response curves were calculated using the Brain-Cousens model, ${ }^{17}$ and performed with $\mathrm{R}^{18}$ using the package drc. ${ }^{19}$

\section{Results}

\section{$\mathrm{H}_{2} \mathrm{O}_{2} / \mathrm{Fe}^{\prime \prime \prime} /$ ascorbic acid system}

Myricetin showed antioxidant effects (Fig. 2A). This effect was stronger in the $\mathrm{FeCl}_{3}$ than in the FeEDTA variant. Within the tested concentration range, $\mathrm{FeCl}_{3}$ followed a full sigmoidal dose-response curve 

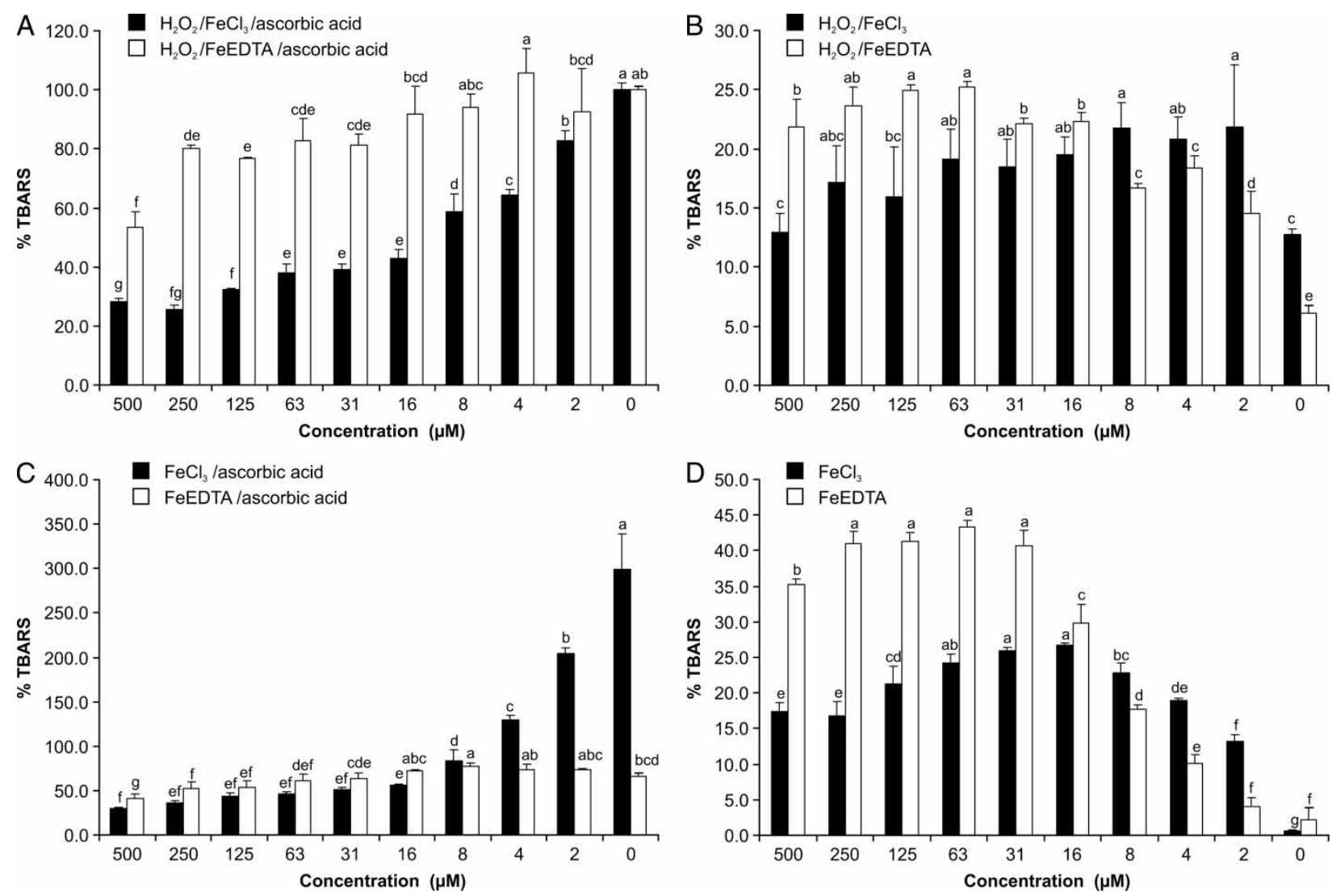

Figure 2 Effects of myricetin on TBARS formation in various systems and variants of the deoxyribose degradation assay; $100 \%$ TBARS represents mean formation in the $\mathrm{FeCl}_{3}$ or FeEDTA variant of the classical ascorbic acid- $\mathrm{H}_{2} \mathrm{O}_{2}$ system; bars are mean \pm $\mathrm{SD} ; \boldsymbol{N}=3$, letters indicate different levels of significance between effects (95\% Duncan's multiple range test).

(Fig. 3A) while FeEDTA only showed a portion of this response (Fig. 3B). The antioxidant effects became more pronounced by increasing the ratio myricetin:Fe.

\section{$\mathrm{H}_{2} \mathrm{O}_{2} / \mathrm{Fe}^{\prime \prime \prime}$ system}

Myricetin was able to replace ascorbic acid in the reaction mixture and start the Fenton reaction in both variants (Fig. 2B). The pro-oxidant effect, however, changed according to the myricetin concentration and resulted in a non-linear U-shaped dose-response curve. This was visible in both variants and again less pronounced in the FeEDTA variant (Figs 3C and D).

\section{$\mathrm{Fe}^{\prime \prime \prime}$ / ascorbic acid system (ascorbic acid autoxidation)}

In the $\mathrm{FeCl}_{3}$ variant, myricetin showed antioxidant effects increasing with its concentration (Fig. 2C). In the FeEDTA variant, it had pro-oxidant activity in lower concentrations and antioxidant effects in higher concentrations. The dose-response curves reflected the contrasting activities of the two variants (Figs $3 \mathrm{E}$ and $\mathrm{F}$ ); an increasing myricetin:Fe ratio further stimulated antioxidant activity if iron was in complex with myricetin. If it was in complex with EDTA, the antioxidative activity was less pronounced.

\section{$\mathrm{Fe}^{\text {III }}$ system (myricetin autoxidation)}

In both variants of the assay, the pro-oxidant effect was clearly visible (Fig. 2D). The highest TBARS concentrations were found in the concentration range of $16-63 \mu \mathrm{M}$ in the $\mathrm{FeCl}_{3}$ variant and $31-250 \mu \mathrm{M}$ in the FeEDTA variant. Higher concentrations, however, decreased the pro-oxidative effect. In both variants, myricetin caused a U-shaped dose-response effect, which was more pronounced in the FeEDTA variant (Figs $3 \mathrm{G}$ and $\mathrm{H}$ ).

\section{Discussion}

All proposed reactions are hypothetical, representing only a portion of the possible ones, which could explain myricetin's anti oxidant and pro-oxidant activities in different chemical environments. Besides ROS scavenging, myricetin's iron chelation ability also affects its antioxidant efficacy - otherwise, the dose-response curves in both iron variants would be identical. This reaction behavior compares with the structurally, closely related flavonoid quercetin, which has one hydroxyl group less on ring B than myricetin, ${ }^{11}$ confirming the notion that flavonoid pyrogallol or catechol moieties are oxidized first to semiquinones or quinones ${ }^{8}$ (Fig. 4). Flavonoids also slow the Fenton reaction by chelating iron ions, which affects the redox potentials of both the ligand 
A $+\mathrm{H}_{2} \mathrm{O}_{2}+$ ascorbic acid

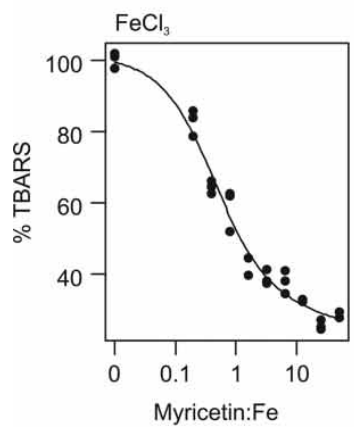

B $+\mathrm{H}_{2} \mathrm{O}_{2}+$ ascorbicacid

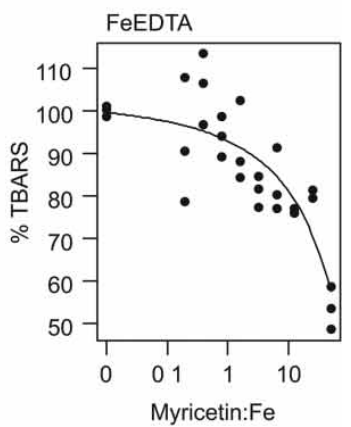

C $+\mathrm{H}_{2} \mathrm{O}_{2}$

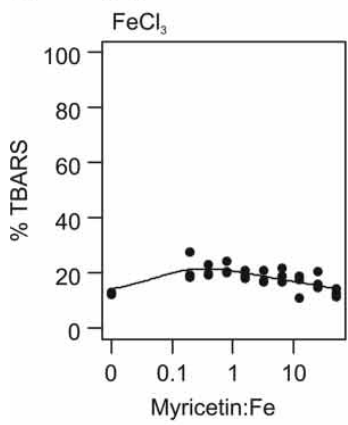

E + ascorbic acid
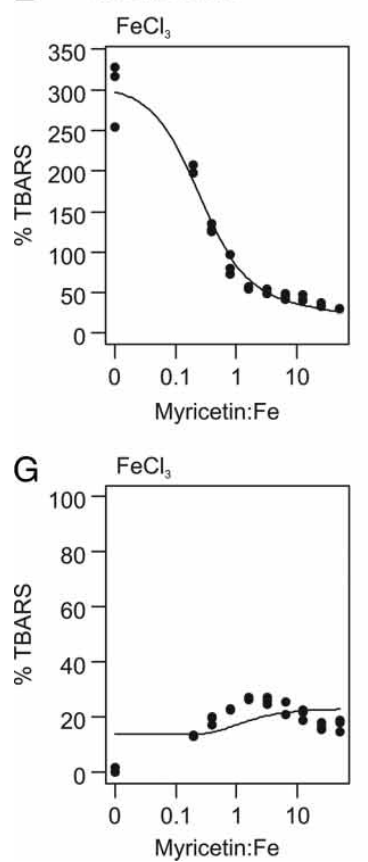

D $+\mathrm{H}_{2} \mathrm{O}_{2}$

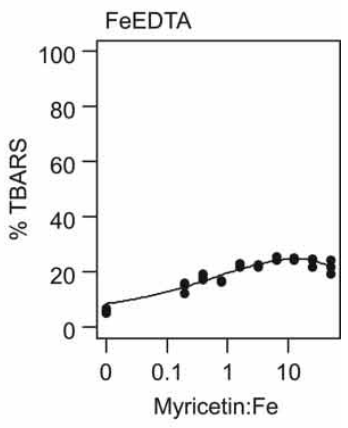

F + asoorbicacid
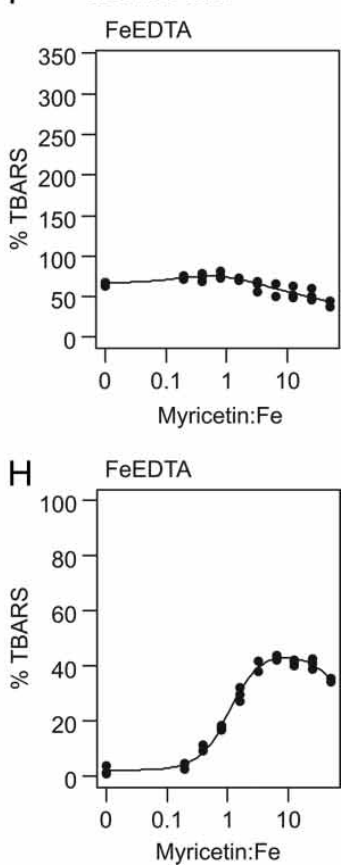

Figure 3 Dose-response curves of TBARS formation depending on the myricetin:Fe ratio in various systems and variants of the deoxyribose degradation assay; $100 \%$ TBARS represents mean formation in the $\mathrm{FeCl}_{3}$ or FeEDTA variant of the classical ascorbic acid- $\mathrm{H}_{2} \mathrm{O}_{2}$ system; $X$-axis has logarithmic scaling but values are not transformed.

and the central atom. ${ }^{20-23}$ Iron-flavonoid complexes are more efficient antioxidant agents than the free flavonoids. $^{22}$ Myricetin-iron(II) complexes have been shown to be stable and to compare with the well-known chelation agent desferoxamine at a neutral $\mathrm{pH} .^{20,24}$ Our experiments illustrate that iron-myricetin complexes are less efficient catalysts of the Fenton

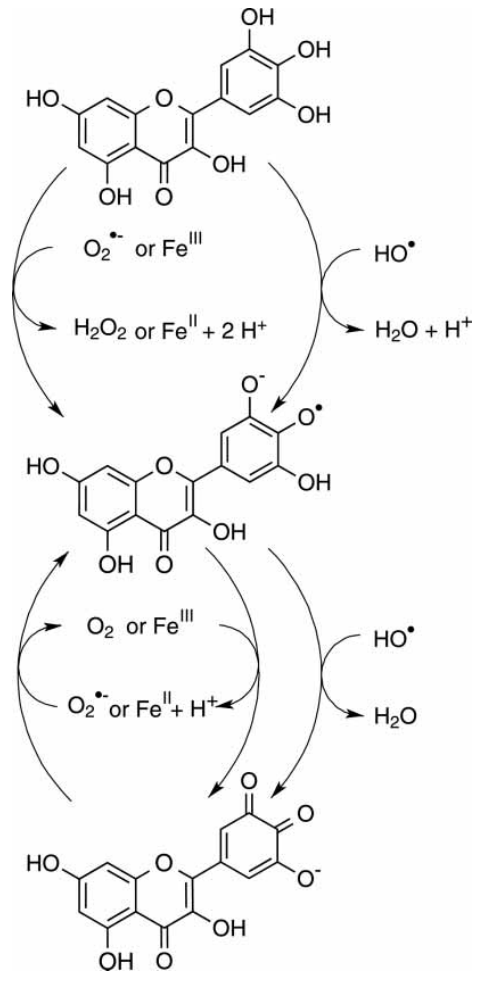

Figure 4 Redox reactions of myricetin.

reaction than iron-EDTA or iron-ascorbic acid complexes. Consequently, we can show that iron complex formation represents a substantial component of myricetin's antioxidative activity.

In the absence of ascorbic acid (Figs 2B, D, 3C, D, $\mathrm{G}$, and $\mathrm{H}$ ), paradoxically, myricetin is a pro-oxidant. This suggests that myricetin is more or less able to substitute for ascorbic acid and reduce iron, molecular oxygen and ROS, albeit less efficiently than ascorbic acid itself (see Fig. 4). Our observations concur with previous literature reports. ${ }^{25,26}$ Myricetin reduced iron(III) to iron(II) in both variants of the assay system, $\mathrm{FeCl}_{3}$ and $\mathrm{FeEDTA}$, starting the Fenton reaction:

Myricetin $-\mathrm{Fe}^{\mathrm{III}}$ complex $\rightarrow$ Myricetin semiquinone $-\mathrm{Fe}^{\mathrm{II}}$ complex

Myricetin + Fe(III)EDTA $\rightarrow$ Myricetin semiquinone

$$
+\mathrm{Fe}(\mathrm{II}) \mathrm{EDTA}
$$

The myricetin semiquinone-iron(II) complex can reduce hydrogen peroxide to hydroxyl radical (Fig. 2B). If iron is in complex with EDTA, myricetin reduces the complex (Fig. 3D). In higher concentration, however, scavenging effects start to prevail due to the larger numbers of myricetin molecules present in the solution. These equilibrium dynamics contribute to a U-shaped dose-response effect (Figs 3C and D).

The pyrogallol structure of the ring B strongly adds to the pro-oxidant activity of myricetin; the related 
catechol flavonoid quercetin was more or less inactive in the identical assay. ${ }^{11}$ Min and Eber reported that myricetin increased calf thymus DNA nucleic base oxidation product concentrations in a hydrogen peroxide/iron(II) system more than quercetin. ${ }^{27}$ These authors interpreted this phenomenon by suggesting that myricetin increases hydrogen peroxide concentration by autoxidation. In contrast, we show here that myricetin's pro-oxidant effect can also be affected by iron(II)/iron(III) redox cycling, probably more than by direct reduction of molecular oxygen. The kinetics of the iron(III) reduction is crucial for Fentontype reactions. ${ }^{28,29}$ In complex with myricetin (or other structurally similar flavonoids), the iron(III) reduction is slowed at a neutral or weakly alkaline $\mathrm{pH}$. Subsequent depletion of iron(II) slows the Fenton reaction. ${ }^{20}$ Our results, which were obtained in specifically developed systems and variants of the deoxyribose degradation assay that aim at exploring the catalytic properties of transition metals on the redox chemistry of test compounds, illustrate that the reduction of iron(III) occurred in complexes with both ligands, EDTA and myricetin. With higher myricetin concentrations, however, the ROS scavenging activity of the pyrogallol moiety again starts to prevail, which inhibits 2-deoxy-D-ribose degradation.

Ascorbic acid is a common plant metabolite and renowned for its antioxidant properties aimed at maintaining the redox homeostasis in the cell. ${ }^{30}$ Conversely, its reducing properties are utilized in the deoxyribose degradation assay to generate hydroxyl radicals from hydrogen peroxide; iron(II) is an important catalyst. The $\mathrm{Fe}^{\mathrm{III}} /$ ascorbic acid system (Fig. 2C) indicates that, if iron is complexed by the strong ligand EDTA, less hydroxyl radicals are formed than if iron is in complex with ascorbic acid. This further illustrates that ligand quality can affect the redox potential of the central iron atoms in a complex. ${ }^{21,31}$ Myricetin probably removes the central atom from the iron-ascorbic acid complex, thus decreasing hydroxyl radical formation by changing the redox potential of iron in the new complex as well as by scavenging ROS; this may explain the six-fold reduction of TBARS levels already at a low-concentration range (Figs 3E and F). While pro-oxidant effects of myricetin, in the presence of ascorbic acid and EDTA, have already been documented, ${ }^{25}$ the non-linearity of this reaction is a novel finding.

The scenario, however, offers even more complexity: Myricetin can reduce iron(III), not only in complex with EDTA, but, in higher concentrations, also in complex with other myricetin molecules $\left(\mathrm{Fe}^{\mathrm{III}}\right.$ system in Fig. 2D). This phenomenon is even better demonstrated in Figs $3 \mathrm{G}$ and $\mathrm{H}$. A higher ratio myricetin:Fe increased TBARS production but in higher concentration myricetin's scavenging properties again became more prominent. In both variants of the $\mathrm{Fe}^{\mathrm{III}}$ system lacking hydrogen peroxide, ROS were generated following myricetin's autoxidation. The electrons were transferred to molecular oxygen giving rise to superoxide anion radicals. Dismutation then led to hydrogen peroxide. In contrast to quercetin, myricetin showed pro-oxidant effects with both $\mathrm{Fe}^{\mathrm{III}}$ variants. The former induced TBARS production only in the FeEDTA variant, and no U-shaped effect was visible at higher concentrations up to the solubility limit. ${ }^{11}$ The pyrogallol arrangement of ring B is probably crucial for iron central atom reduction in the flavonoid complex in a weakly alkaline $\mathrm{pH}$. An electron paramagnetic resonance study ${ }^{20}$ identified a semiquinone signal of the autoxidized myricetin after iron ion addition at a weakly alkaline $\mathrm{pH} .{ }^{20}$ In agreement with our results, two studies have reported autoxidation of myricetin in the presence of iron ions and subsequent ROS production. ${ }^{25,32}$ ROS were formed via molecular oxygen reduction to superoxide anion radical. $^{33}$

\section{Conclusion}

The systems and variants of the deoxyribose degradation assay allow substantial insights into the autoxidation dynamics of the pyrogallol moiety of myricetin. It increases the reducing power of the flavonoid, which also can result in pro-oxidant activity. Firstly, liganded iron(III) is reduced to iron(II). The autoxidation of iron(II) transfers electrons to molecular oxygen, resulting in the formation of superoxide anion radicals and other ROS. Myricetin's pro- and/or antioxidant effects depend on both the concentration of the reactants and reaction conditions. The final outcome is determined by many reversible and simultaneous reactions as evidenced by the U-shaped dose-response curves.

\section{Acknowledgements}

This research was supported by a Lise Meitner fellowship of the Austrian Science Fund (FWF M920-B03). The authors are indebted to Prof. Sharon Robinson (University of Wollongong, Wollongong, Australia) for linguistic corrections.

\section{References}

1 Ross JA, Kasum CM. Dietrary flavonoids: Bioavailability, metabolic effects, and safety. Annu Rev Nutr 2002;22(1):19-34.

2 Buer CS, Imin N, Djordjevic MA. Flavonoids: new roles for old molecules. J Integr Plant Biol 2010;52(1):98-111.

3 Hadacek F, Bachmann G, Egelmeier D, Chobot V. Hormesis and a chemical raison d'être for secondary plant metabolites. Dose-Response 2011;9(1):79-116.

4 Chobot V, Huber C, Trettenhahn G, Hadacek F. ( \pm )-Catechin: chemical weapon, antioxidant, or stress regulator? J Chem Ecol 2009;35(8):980-96.

5 Halliwell B. Reactive species and antioxidants. Redox biology is a fundamental theme of aerobic life. Plant Physiol 2006;141(2): 312-22. 
6 Foyer $\mathrm{CH}$, Noctor G. Oxidant and antioxidant signalling in plants: a re-evaluation of the concept of oxidative stress in a physiological context. Plant Cell Environ 2005;28(8):1056-71.

7 Chobot V, Hadacek F. Milieu-dependent pro- and antioxidant activity of juglone may explain linear and nonlinear effects on seedling development. J Chem Ecol 2009;35(3):383-90.

8 Rice-Evans CA, Miller NJ, Paganga G. Structure-antioxidant activity relationships of flavonoids and phenolic acids. Free Radic Biol Med 1996;20(7):933-56.

9 Prochazkova D, Bousova I, Wilhelmova N. Antioxidant and prooxidant properties of flavonoids. Fitoterapia 2011;82(4) 513-23.

10 Halliwell B. Are polyphenols antioxidants or pro-oxidants? What do we learn from cell culture and in vivo studies? Arch Biochem Biophys 2008;476(2):107-12

11 Chobot V. Simultaneous detection of pro- and antioxidative effects in the variants of the deoxyribose degradation assay. J Agric Food Chem 2010;58(4):2088-94.

12 Ong KC, Khoo H-E. Biological effects of myricetin. Gen Pharmacol 1997;29(2):121-6.

13 Aruoma OI, Grootveld M, Halliwell B. The role of iron in ascorbate-dependent deoxyribose degradation - evidence consistent with a site-specific hydroxyl radical generation caused by iron ions bound to deoxyribose molecule. J Inorg Biochem 1987; 29(4):289-99.

14 Halliwell B, Gutteridge JMC, Aruoma OI. The deoxyribose method - a simple test-tube assay for determination of rate constants for reactions of hydroxyl radicals. Anal Biochem 1987; 165(1):215-9.

15 Aruoma OI. Assessment of potential prooxidant and antioxidant actions. J Am Oil Chem Soc 1996;73(12):1617-25.

16 Buettner GR. In the absence of catalytic metals ascorbate does not autoxidize at $\mathrm{pH} 7$ : ascorbate as a test for catalytic metals. J Biochem Biophys Methods 1988;16(1):27-40.

17 Brain P, Cousens R. An equation to describe dose responses where there is stimulation of growth at low doses. Weed Res 1989;29(2):93-6.

18 Team RDC. R: A language and environment for statistical computing. Vienna, Austria: $\mathrm{R}$ Foundation for Statistical Computing; 2011.

19 Ritz C, Streibig JC. Bioassay analysis using R. J Stat Softw 2005; 12(5):1-22.

20 Perron NR, Brumaghim JL. A review of the antioxidant mechanisms of polyphenol compounds related to iron binding. Cell Biochem Biophys 2009;53(2):75-100.
21 Chobot V, Hadacek F. Iron and its complexation by phenolic cellular metabolites: from oxidative stress to chemical weapons. Plant Signal Behav 2010;5(1):4-8.

22 de Souza RFV, De Giovani WF. Antioxidant properties of complexes of flavonoids with metal ions. Redox Rep 2004;9(2):97-104.

23 Miller DM, Buettner GR, Aust SD. Transition metals as catalysts of autoxidation reactions. Free Radic Biol Med 1990;8(1): 95-108.

24 Mladenka P, Macakova K, Filipsky T, Zatloukalova L, Jahodar $\mathrm{L}$, Bovicelli $\mathrm{P}$, et al. In vitro analysis of iron chelating activity of flavonoids. J Inorg Biochem 2011;105(5):693-701.

25 Laughton MJ, Halliwell B, Evans PJ, Hoult JRS. Antioxidant and pro-oxidant actions of the plant phenolics quercetin, gossypol and myricerin. Effects on lipid-peroxidation, hydroxyl radical generation and bleomycin-dependent damage to DNA. Biochem Pharmacol 1989;38(17):2859-65.

26 Mira L, Fernandez MT, Santos M, Rocha R, Florencio MH, Jennings KR. Interactions of flavonoids with iron and copper ions: a mechanism for their antioxidant activity. Free Radic Res 2002;36(11):1199-208.

27 Min K, Ebeler SE. Flavonoid effects on DNA oxidation at low concentrations relevant to physiological levels. Food Chem Toxicol 2008;46(1):96-104.

28 Buettner GR, Doherty TP, Patterson LK. The kinetics of the reaction of superoxide radical with $\mathrm{Fe}(\mathrm{III})$ complexes of EDTA, DETAPAC and HEDTA. FEBS Lett 1983;158(1): 143-6.

29 Cheng ZY, Li YZ, Chang WB. Kinetic deoxyribose degradation assay and its application in assessing the antioxidant activities of phenolic compounds in a Fenton-type reaction system. Anal Chim Acta 2003;478(1):129-37

30 Davey MW, Van Montagu M, Inze D, Sanmartin M, Kanellis A, Smirnoff N, et al. Plant L-ascorbic acid: chemistry, function, metabolism, bioavailability and effects of processing. J Sci Food Agric 2000;80(7):825-60

31 Kell DB. Iron behaving badly: inappropriate iron chelation as a major contributor to the aetiology of vascular and other progressive inflammatory and degenerative diseases. BMC Med Genom. 2009;2(Article No. 2).

32 Canada AT, Giannella E, Nguyen TD, Mason RP. The production of reactive oxygen species by dietary flavonoids. Free Radic Biol Med 1990;9(5):441-9.

33 Miura YH, Tomita I, Watanabe T, Hirayama T, Fukui S. Active oxygens generation by flavonoids. Biol Pharm Bull 1998;21(2): 93-6. 